\title{
GABAergic Neurons of the Medial Septum Lead the Hippocampal Network during Theta Activity
}

\author{
Balázs Hangya, ${ }^{1}$ Zsolt Borhegyi, ${ }^{1}$ Nóra Szilágyi, ${ }^{2}$ Tamás F. Freund, ${ }^{1}$ and Viktor Varga ${ }^{1}$ \\ ${ }^{1}$ Laboratory of Cerebral Cortex Research, Department of Cellular and Network Neurobiology, Institute of Experimental Medicine, Hungarian Academy of \\ Sciences, H-1083 Budapest, Hungary, and ²Department of Physiology and Neurobiology, Eötvös Loránd University, H-1117 Budapest, Hungary
}

Information processing in the hippocampus critically relies on its reciprocal interaction with the medial septum (MS). Synchronization of the septo-hippocampal system was demonstrated during both major hippocampal activity states, the regular theta rhythm and the large amplitude irregular activity. Previous experimental and modeling data suggest that the MS provides rhythmic drive to the hippocampus, and hippocampo-septal feedback synchronizes septal pacemaker units. However, this view has recently been questioned based on the possibility of intrahippocampal theta genesis. Previously, we identified putative pacemaker neurons expressing parvalbu$\min (\mathrm{PV})$ and/or the pacemaker hyperpolarization-activated and cyclic nucleotide-gated nonselective cation channel (HCN) in the MS. In this study, by analyzing the temporal relationship of activity between the PV/HCN-containing medial septal neurons and hippocampal local field potential, we aimed to uncover whether the sequence of events during theta formation supports the classic view of septal drive or the challenging theory of hippocampal pacing of theta. Importantly, by implementing a circular statistical method, a temporal lead of these septal neurons over the hippocampus was observed on the course of theta synchronization. Moreover, the activity of putative hippocampal interneurons also preceded hippocampal local field theta, but by a shorter time period compared with PV/HCN-containing septal neurons. Using the concept of mutual information, the action potential series of PV/HCN-containing neurons shared higher amount of information with hippocampal field oscillation than $\mathrm{PV} / \mathrm{HCN}$-immunonegative cells. Thus, a pacemaker neuron population of the MS leads hippocampal activity, presumably via the synchronization of hippocampal interneurons.

\section{Introduction}

The hippocampus exhibits two dominant, behavioral statedependent types of local field potential (LFP) patterns, the 4-10 $\mathrm{Hz}$ theta rhythm and the large amplitude irregular activity (LIA) with sharp waves (Buzsáki et al., 1983). The medial septum (MS) is an indispensable component of the theta-generating subcortical network (Lawson and Bland, 1993; Vertes and Kocsis, 1997). However, the hippocampo-septal backprojection has recently been proved to be crucial for synchronizing the medial septal and hippocampal circuits (Tóth et al., 1993; Wang, 2002). Furthermore, the classic hypothesis of MS drive to hippocampal theta has been challenged by Manseau et al. (2008), suggesting a mechanism of intrahippocampal theta genesis. Thus, in this study, we investigated the following possible scenarios: (1) putative pacemaker MS neurons lead hippocampal field theta and hippocampal interneurons, proving a pacemaker role for the MS; (2) the activity changes of hippocampal interneurons precede those of MS cells, supporting the theory of hippocampal pacing of theta;

\footnotetext{
Received Nov. 20, 2008; revised April 8, 2009; accepted May 19, 2009.

This work was supported by Országos Tudományos Kutatási Alapprogramok Grant K60927. We thank Ryuich Shigemoto for providing the HCN1 and HCN2 antibodies and Drs. Szabolcs Káli, László Acsády, Gábor Nyíri, and Máté Lengyel for helping us to prepare this manuscript. We thank Katalin Iványi, Zoltánné Lengyel, Emőke Szépné Simon, and Győző Goda for the excellent technical assistance.

Correspondence should be addressed to Balázs Hangya, Laboratory of Cerebral Cortex Research, Department of Cellular and Network Neurobiology, Institute of Experimental Medicine, Hungarian Academy of Sciences, Szigony utca 43, H-1083 Budapest, Hungary. E-mail: hangyab@koki.hu.

D01:10.1523/JNEUROSCI.5665-08.2009

Copyright $\odot 2009$ Society for Neuroscience $\quad$ 0270-6474/09/298094-09\$15.00/0
}

(3) no significant temporal difference can be observed, which raises the possibility of a septal and a hippocampal pacemaker alternating or operating coincidentally, or a potential hidden pacemaker synchronizing the two areas. By implementing the circular statistical $Z$-shift method (Siapas et al., 2005), we demonstrated that alterations in the activity of the previously identified candidate pacemaker neurons (Borhegyi et al., 2004; Varga et al., 2008) in the MS precede those of putative hippocampal interneurons as well as corresponding changes in hippocampal field theta. Then, by using an information theoretical approach based on mutual information (MI) (Panzeri et al., 2007), we could show that the candidate pacemaker population of $\mathrm{PV} / \mathrm{HCN}$ neurons share a higher amount of information with hippocampal theta than PV/HCN-immunonegative cells. Our data demonstrate that a subpopulation of MS GABAergic neurons serves as pacemakers of hippocampal theta, transmitting rhythmic activity from the MS to the hippocampus.

\section{Materials and Methods}

In this study, partly the same datasets were analyzed as those published in the studies by Borhegyi et al. (2004) and Varga et al. (2008) (supplemental Table 1, available at www.jneurosci.org as supplemental material). Thus, only a brief description of electrophysiological and anatomical methods will be provided here (for full details, see the cited references and supplemental Methods, available at www.jneurosci.org as supplemental material).

\section{Animal handling}

Male Wistar rats of 200-400 g body weight were used in the present study (Charles River; or bred in the own dedicated specific pathogen-free 
animal facility of the Institute). Experiments were performed according to the guidelines of the Institutional Ethical Codex and the Hungarian Act of Animal Care and Experimentation (1998, XXVIII, section 243/ 1998), which conforms to the regulation of animal experiments by the European Union. Animals were kept under a $12 \mathrm{~h}$ light/dark cycle; water and food was available ad libitum. All efforts were made to minimize pain and suffering and to reduce the number of animals used.

\section{Data recording}

Rats were urethane anesthetized. Wide-band $(0.3 \mathrm{~Hz}$ and 2 or $5 \mathrm{kHz})$ hippocampal LFP was recorded monopolarly from the CA1 pyramidal layer and digitized at $10 \mathrm{kHz}$. Medial septal or hippocampal unit activity was recorded concurrently with hippocampal LFP by the juxtacellular technique. Single-cell activity was amplified, filtered $(0.1$ and $5 \mathrm{kHz})$, and then digitized at $10 \mathrm{kHz}$. Hippocampal theta was elicited by sensory stimulation (tail pinch) and/or occurred spontaneously. The recording period was followed by juxtacellular biocytin or Neurobiotin labeling of the neuron.

\section{Identification of juxtacellularly labeled neurons}

After data recording, labeling, and a survival period of 10-120 min, the animals were perfused transcardially. Brains were removed and the block containing the septum was cut and postfixed overnight. Coronal sections of $60 \mu \mathrm{m}$ thick were cut on a vibratome from the tissue blocks. The immunofluorescent identification of neurons with the list of used primary and secondary antibodies is described by Borhegyi et al. (2004) and Varga et al. (2008) and also in the supplemental material (available at www.jneurosci.org). HCN1-immunoreactive neurons are referred to as $\mathrm{HCN}$ immunoreactive throughout this paper. HCN1 and HCN2 were shown to completely colocalize in the MS (Varga et al., 2008). Representative examples of labeled medial septal neurons are shown in Figure 3.

\section{Data analysis}

Wavelet spectrum. Our signals recorded in time-domain were decomposed in time-frequency domain using CWT (continuous wavelet transform), as published by Torrence and Compo (1998) (for a brief description, see also Borhegyi et al., 2004). Medial septal unit recording was first discriminated (i.e., converted into a $0-1$ series with 1 at the localization of spikes and 0 elsewhere) and convolved with a $\operatorname{sinc}[\sin (x) / x]$ kernel before wavelet transformation. Hippocampal LFP and sinc-convolved units were downsampled on $1000 \mathrm{~Hz}$, standardized (normalized to zero mean and unitary SD), and then decomposed to 189 scales with equivalent Fourier frequencies varying from 0.5 to $500 \mathrm{~Hz}$, which corresponded to the Nyquist frequency (see Fig. $2 B$ ).

Selection of analyzed segments. Theta segments were defined as episodes in which wavelet power spectral maxima of the hippocampal LFP fell in the theta band [which was chosen between 2.5 and $6 \mathrm{~Hz}$ because of the observation that urethane anesthesia lowers the limits of the traditional frequency bands (Leung, 1985; Clement et al., 2008)]. Sharp wave segments were detected after filtering the LFP between 90 and $145 \mathrm{~Hz}$ (Klausberger et al., 2003) using high-order finite impulse response filters, and calculating the root mean square (RMS) of the filtered signal in 100 point nonoverlapping windows. The detection threshold for ripples was mean +5 SDs of the RMS of the signal; the beginning and end of ripples were determined at RMS values of mean +1 SD. Segments of LIA were defined as nontheta nonsharp wave segments; they are referred as "nontheta segments" hereinafter. The 200-ms-long edges were left from the beginning and the end of all theta and nontheta segments. Only segments $>5 \mathrm{~s}$ were taken into account.

Identification of theta-bursting medial septal neurons and hippocampal interneurons. To separate septal neurons showing bursting activity pattern with frequency of bursts falling in the theta range (i.e., thetabursting neurons), we applied the following semiautomatic procedure. Hierarchic cluster analysis on interspike intervals using Euclidean distance measure and Ward's amalgamation rule was performed (Dekhuijzen and Bagust, 1996). Cluster tree (dendrogram) was cut iteratively at different linkage distances to have two to seven clusters, and the cluster containing the smallest interspike interval (which obviously corresponded to the cluster of intraburst intervals for bursting neurons) was selected at each case. For each iteration, a recurrence plot was produced showing every interspike interval against the previous interspike interval. If any of the iterated clustering processes succeeded to separate the intraburst intervals (as cluster containing the smallest interval) on the recurrence plot, we considered the neuron to be theta-bursting.

Hippocampal interneurons were differentiated from principal cells based on the following assumptions: interneurons fire at higher rate [mean firing rate, $6.52 \pm 6.21 \mathrm{~Hz}$ (mean $\pm \mathrm{SD}$ ); peak firing rate calculated in $5 \mathrm{~s}$ nonoverlapping windows, $10.78 \pm 8.37 \mathrm{~Hz}$ ], produce narrower spikes (width at maximal amplitude, $0.58 \pm 0.37 \mathrm{~ms}$ ), and did not fire complex spikes (equivalent to low-threshold bursts) (Csicsvari et al., 1999; Fuentealba et al., 2008).

Z-shift methods. Phase values for medial septal action potentials as correlated to hippocampal LFP were defined using the Hilbert transform (analytic signal method) [for detailed formulation, see, for example, Hurtado et al. (2004)].

Time delay between MS unit and hippocampal LFP was determined using the phase-locking analysis of time-shifted signals published first in the study by Siapas et al. (2005). The algorithm is based on Rayleigh's $Z$-statistic and is referred to here as " $Z$-shift analysis." See Figure 1 and supplemental Figure 1 (available at www.jneurosci.org as supplemental material) for an illustration of the procedure on a medial septal and on a hippocampal neuron. The null hypothesis of Rayleigh's test is that phase angle values show uniform distribution on the circle, whereas the alternative hypothesis stands for phase preference (i.e., unimodal nonuniform phase angle distribution) (Fisher, 1993). In our case, the value of Rayleigh's $Z$-statistic indicates strength of phase coupling (or degree of nonuniformity) between unit events and hippocampal field potential. After fixing hippocampal LFP in the time domain, the unit was shifted relative to the field activity by different $\tau$ (time) values $(-1 \mathrm{~s}<\tau<1 \mathrm{~s}$ ). Subsequently, we calculated Rayleigh's $Z$-statistics (proportion to degree of phase-locking) for all time shifts $(\tau)$. $Z$-statistics are given by the following equation:

$$
Z=n \bar{R}^{2},
$$

where $R$ denotes the mean resultant length of the given phase series $\Phi_{j}$ $(j=1, \ldots, n)$ :

$$
\bar{R}=\left|\frac{1}{n} \sum_{j=1}^{n} e^{j \phi_{j}}\right| .
$$

The probability that the null hypothesis of uniformity holds can be calculated as follows:

$$
p=e^{-z}\left[1+\left(2 Z-Z^{2}\right) /(4 n)-\left(24 Z-132 Z^{2}+76 Z^{3}-9 Z^{4}\right) /\left(288 n^{2}\right)\right]
$$

for $n \geq 50, p=e^{-Z}$ approximation is appropriate. (However, the exact $p$ value was calculated in the present study in all cases, regardless of the value of $n$.) $Z$-shift was defined as the $\tau$ value by which shifting unit relative to LFP results in maximum degree of phase-locking (the highest value of the $Z$-statistics) (Fig. 1). In Figure $1 C$, the highest peak of the $Z$ value in the function of time lags corresponds to strongest phase coupling. It appears in the positive halfplane, which implies that the action potential series have to be shifted with a positive value to get maximal phase preference; thus unit leads LFP activity. Vice versa, highest peak in the negative halfplane would infer the lead of the LFP signal over unit activity. Medial septal or hippocampal units that failed to reach a significant level of phase preference at any delay values ( $p>0.005$, Rayleigh's test) were excluded from this analysis. Thus, $Z$-shift algorithm is applicable for units with significant phase preference (at least after the application of a given delay) [i.e., for theta-bursting cells or neurons showing frequency changes at specific phases of the theta cycle ("theta-modulated cells")]. However, it is inappropriate for neurons with theta-independent firing pattern (e.g., for tonic or irregularly firing cells). Using this method, we were able to determine the time delay between corresponding events in the medial septal or hippocampal unit and hippocampal field activity (Siapas et al., 2005). Comparisons between anatomical groups were performed with the Mann-Whitney $U$ test. 

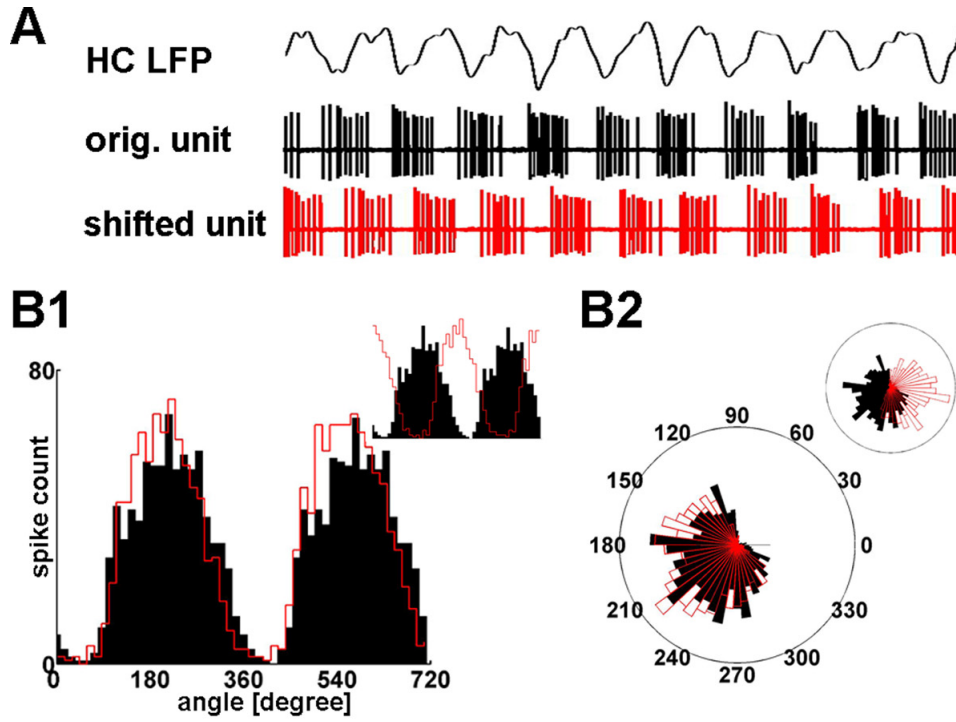

Figure 1. Z-shift methods. A, Z-shift analysis of the longest theta segment of a registration is demonstrated. A 5-s-long epoch from the hippocampal LFP bandpass filtered between 2 and 8 Hz is shown above medial septal unit activity. Red action potential series correspond to the MS unit delayed by Z-shift, the optimal time lag with which shifting unit activity results in the strongest phase locking (see Materials and Methods). B1, Circular distribution of phase values of septal action potentials relative to hippocampal theta oscillation is displayed in black for two theta cycles (zero phase was associated to the trough of the theta cycle). The red line indicates phase angle distribution of the shifted septal action potentials, centered to overlap with the original distribution. (The relationship of the two histograms without centering is shown on the inset.) Histogram of shifted unit is slightly more focused than the original, which means higher level of phase preference. Rayleigh's Z-statistic is sensitive enough to measure such mild differences reliably. B2, The same phase histograms are shown on rose diagram; inset displays rose diagram without centering the histogram of shifted action potentials. C, Rayleigh's Z-statistic is plotted against different time lags from -1 to $1 \mathrm{~s}$. The red line shows the level of significant phase locking at $p=0.005$. Maximum location of the function is Z-shift, the optimal delay to reach the highest "synchrony" between LFP and septal unit, whereas maximal $Z$ value describes the strength of the maximal coupling.

Information theoretical approach. After calculating wavelet spectra of both signals, wavelet power matrices were cut into 1-s-long segments for scales from 2.5 to $6 \mathrm{~Hz}$ to get time-frequency windows. Distribution of wavelet power values falling into a specified window was estimated with a histogram. Entropy of the unit and LFP wavelet power distributions was defined using Shannon's formula (Shannon, 1948) as follows:

$$
H(X)=-\sum_{i} p_{i} \log p_{i}
$$

where $H(X)$ denotes the entropy (or information content) of power value distribution, and $p_{i}$ denotes the relative frequency of the $i$ th bin (Fig. 2C). The same method was used to compute the entropy of the joint power value distribution of corresponding windows of the two signals (Fig. 2D, left).

$$
I(X, Y)=H(X)+H(Y)-H(X, Y) .
$$

Equation 5 gives mutual information $[I(X, Y)]$, where $H(X)$ and $H(Y)$ are the entropies of power value distributions in corresponding windows, $H(X, Y)$ is the entropy of the appropriate joint distribution (Fig. $2 D$, right). The estimation of entropy and mutual information from histograms can carry certain types of bias. Hence these estimations were adjusted using the Panzeri-Treves bias correction method as reported by Panzeri et al. (2007). Balanced pooled samples for group statistics were generated by taking the first four MI values of the analyzed segments. Statistical comparisons were accomplished using either the Mann-Whitney $U$ test (comparison between anatomical or physiological groups of cells) or Wilcoxon's signed rank test (comparison between real and control data or theta and nontheta segments). Theta-associated changes of MI were judged at the level of $p=0.01$ using the Mann-Whitney $U$ test.

For the mutual information calculations, three types of controls were used: original hippocampal LFP was compared with (1) an artificial unit generated by a random Poisson process, (2) original unit with shuffled segments, (3) original unit with shuffled interspike intervals. The first type of control was demonstrated in the main text. (For additional information, see supplemental material, available at www.jneurosci.org.) Significant difference between real and control data was accepted at the level of $p=0.01$.
The analysis was implemented in Matlab development environment (MathWorks) using self-developed and built-in functions. Freely available wavelet program of Christopher Torrence and Gilbert P. Compo (University of Colorado, Boulder, CO) was used for wavelet analysis.

\section{Results}

Time delay between medial septal PV/HCN-immunoreactive neurons and hippocampal LFP suggests septal lead

It is known that, in synchronously operating interconnected neuronal networks, changes in the activity of one network could appear as the perturbation of the other network after a time delay. Temporal difference between correlated alterations of activity may provide new insight into the functional architecture of the septohippocampal connection. Thus, we implemented the recently documented $Z$-shift method to unravel the temporal order in the septohippocampal system during theta activity. In case a neuron directly contributes to the LFP recorded from the same localization, one can observe a strong phase coupling (high $Z$ value) (Fig. 1) of the unit to the concurrent LFP. However, when a neuron contributes to a field oscillation in a distant area, the maximal value of phase-coupling strength appears between the current unit and a later period of the LFP. This delay indicates the time needed for the changes in the unit activity to be reflected in corresponding changes in the LFP. Conversely, if a neuron is affected by a field oscillation in another area, maximal phase locking appears between the current unit and a previous epoch of the LFP. $Z$-shift expresses the time difference by which alterations of hippocampal field oscillation (e.g., minor perturbations) follow $(Z$-shift $>0)$ or precede $(Z$-shift $<0)$ the corresponding minor changes of septal unit activity. Recordings in which the longest theta segment was $<5$ s or the maximal phase coupling was not significant ( $p>0.005$, Rayleigh's test) were excluded, which resulted in the analysis of the longest theta segments of 189 septal neurons (supplemental Table 1, available at www.jneurosci.org as supplemental material). First, it was shown that $Z$-shift is mostly independent from the main statistical parameters (i.e., mean angle 

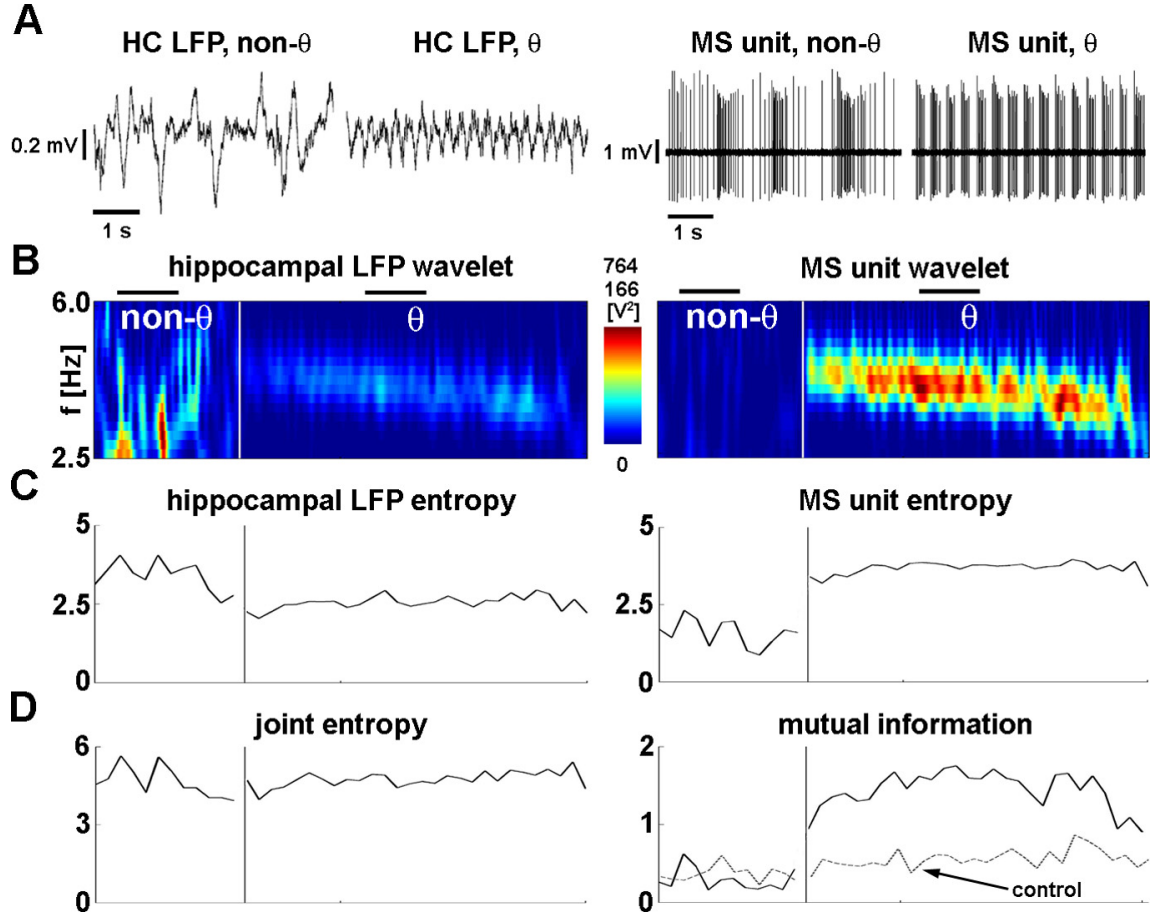

Figure 2. Calculation of wavelet mutual information. $\boldsymbol{A}$, The 5-s-long recordings of hippocampal LFP (left column) and media septal unit (right column). Within the columns, left recordings show nontheta activity, and right recordings correspond to theta oscillation. The demonstrated septal neuron shows theta frequency bursting during theta rhythm. $\boldsymbol{B}$, Wavelet power spectra of the hippocampal LFP (left) and septal unit activity (right). Time is displayed on $x$-axis, whereas frequency is showed on $y$ (only theta band is displayed). Color-coded values indicate the wavelet power coefficients at given time and frequency. Color code is given on the color bar between the two plots; the top value above the color bar shows the upper limit for the LFP, and the bottom value shows the upper limit for unit wavelet power. The white vertical line separates nontheta and theta episodes; the segments before and after the white line are the longest nontheta and theta segments, respectively. The horizontal black lines above the LFP wavelet designate the positions of the 5 -s-long raw data segments shown in $A$. At the onset of theta, the distribution of spectral components markedly changed in both LFP and unit activity: power density concentrated in the theta band. Note that, even during nontheta, significant amount of power can be observed in the theta band (warm color, high power). C, Information content (entropy) of hippocampal LFP (left) and medial septal unit (right). D, Joint information content of LFP and unit (joint entropy, left) and amount of common information (mutual information, right). The dashed line shows control mutual information. At the onset of theta oscillation, (1) theta frequency range information content of the LFP decreased, (2) information content of unit increased, (3) the joint entropy remained relatively unchanged, and (4) the mutual information increased in the presented recording. HC, Hippocampus.

and concentration parameter) determining a circular sample (see supplemental material, available at www.jneurosci.org).

Most of the MS neurons (117 of 189; 61.9\%; 72 of 117 thetabursting) fell between 0 and $200 \mathrm{~ms}$ ( $<0 \mathrm{~ms}$ : 50 of 189, 26.5\%, 23 of 50 theta-bursting; $>200$ ms: 22 of 189, 11.6\%, 8 of 22 theta-bursting; median for all 189 neurons, $58 \mathrm{~ms}$; interquartile range, -1.75 to $129.75 \mathrm{~ms}$ ), especially those with higher maximal $Z$ values. The latter indicates stronger phase coupling and longer distance from the $x$-axis (see Fig. 4). The above finding implies that events during theta oscillations in the activity of septal neurons precede those of the hippocampal field with a median of $58 \mathrm{~ms}$, especially for highly regular, strongly theta phase-locked MS cells. PV-immunoreactive (PV-IR) neurons (25 of 189; 12 of 25 immunoreactive for HCN) (Fig. 3) showed a median delay of $79 \mathrm{~ms}$ (Table 1), which was significantly different from zero $\left(p=4.424 \times 10^{-27}\right)$ and from the delay in PV-immunonegative MS cells $(p=0.0168)$ (Fig. $4 A)$. Median delay of PV-immunonegative neurons (16 of 189) was $11.5 \mathrm{~ms}$, which was not significantly different from zero $(p=0.168)$. Thus, only the MS cells containing PV preceded hippocampal LFP. Additionally, PV-IR neurons formed a more consistent group with a significantly smaller SD (PV-IR SD, $82.10 \mathrm{~ms}$; PV-immunonegative $\mathrm{SD}, 304.60 \mathrm{~ms} ; p=5.226 \times 10^{-8}, F$ test $)$ of the individual delays.
Similar to PV-IR neurons, $\mathrm{HCN}$ immunoreactive (HCN-IR) cells (20 of 189; 12 of 20 immunoreactive for PV) were characterized by a longer delay with a median identical with PV-IR cells as opposed to $\mathrm{HCN}$-immunonegative MS neurons (7 of 189) (HCN-IR median, 79 ms; HCNimmunonegative median, $-78 \mathrm{~ms} ; p=$ 0.005904) (Fig. $4 B$ ). Both medians were significantly different from zero $(p=6.146 \times$ $10^{-6}$ and $p=0.0084$ for $\mathrm{HCN}$ immunopositive and -immunonegative groups, respectively); thus, HCN-IR neurons preceded hippocampal theta, whereas HCN-immunonegative cells followed hippocampal LFP. SD in the HCN-IR group was significantly smaller than in the $\mathrm{HCN}$ immunonegative group (HCN-IR SD, 82.20 ms; HCN-immunonegative SD, 454.49 ms; $p=1.752 \times 10^{-8}, F$ test). Both PV-IR and HCN-IR neurons were better phase locked to hippocampal theta than the PV- or HCNimmunonegative cells showed by the higher maximal $Z$ values on Figure 4. Furthermore, phase modulation was markedly small in the latter cell group, suggesting that strength of phase coupling distinguishes $\mathrm{HCN}$-immunoreactive and $\mathrm{HCN}$ immunonegative cells. The above results are strengthened by the observation that, at the population level, the same results were obtained regardless of analyzing all theta segments of all neurons (supplemental Fig. 4, available at www.jneurosci.org as supplemental material), or only the longest theta segments of septal neurons (Fig. 4), or theta segments with maximal $Z$ value (the strongest phase-coupling) (supplemental Fig. 3, available at www.jneurosci.org as supplemental material). The antecedence of PV/ HCN-IR neuron firing relative to hippocampal LFP activity indicates that changes in the spiking of these neurons may influence theta rhythm in the hippocampus.

To compare the relationship of medial septal neurons and hippocampal interneurons to hippocampal local field oscillation, $Z$-shift was calculated for 27 putative hippocampal interneurons (5 of 27 theta-bursting) (Fig. $4 A, B$ ). These cells preceded hippocampal theta oscillation by a median of $47 \mathrm{~ms}$. Thus, the delay between hippocampal interneurons and LFP was $32 \mathrm{~ms}$ smaller than the delay between PV- or HCN-IR MS cells and hippocampal LFP. This finding substantiates the results of MS unit-hippocampal LFP $Z$-shift analysis and points to an MS-hippocampus direction of influence during theta activity.

\section{Synchronization of PV-and HCN-IR MS neurons to} hippocampal theta oscillation

Common information content (MI) between MS unit and hippocampal LFP in the theta band reflects the strength of theta band synchrony between the two signals. Beyond linear correlation, MI is capable of detecting nonlinear connections that are common among neural structures (Freiwald et al., 1999) and are underestimated or even not detected by linear methods. To unravel whether putative pacemaker neurons 

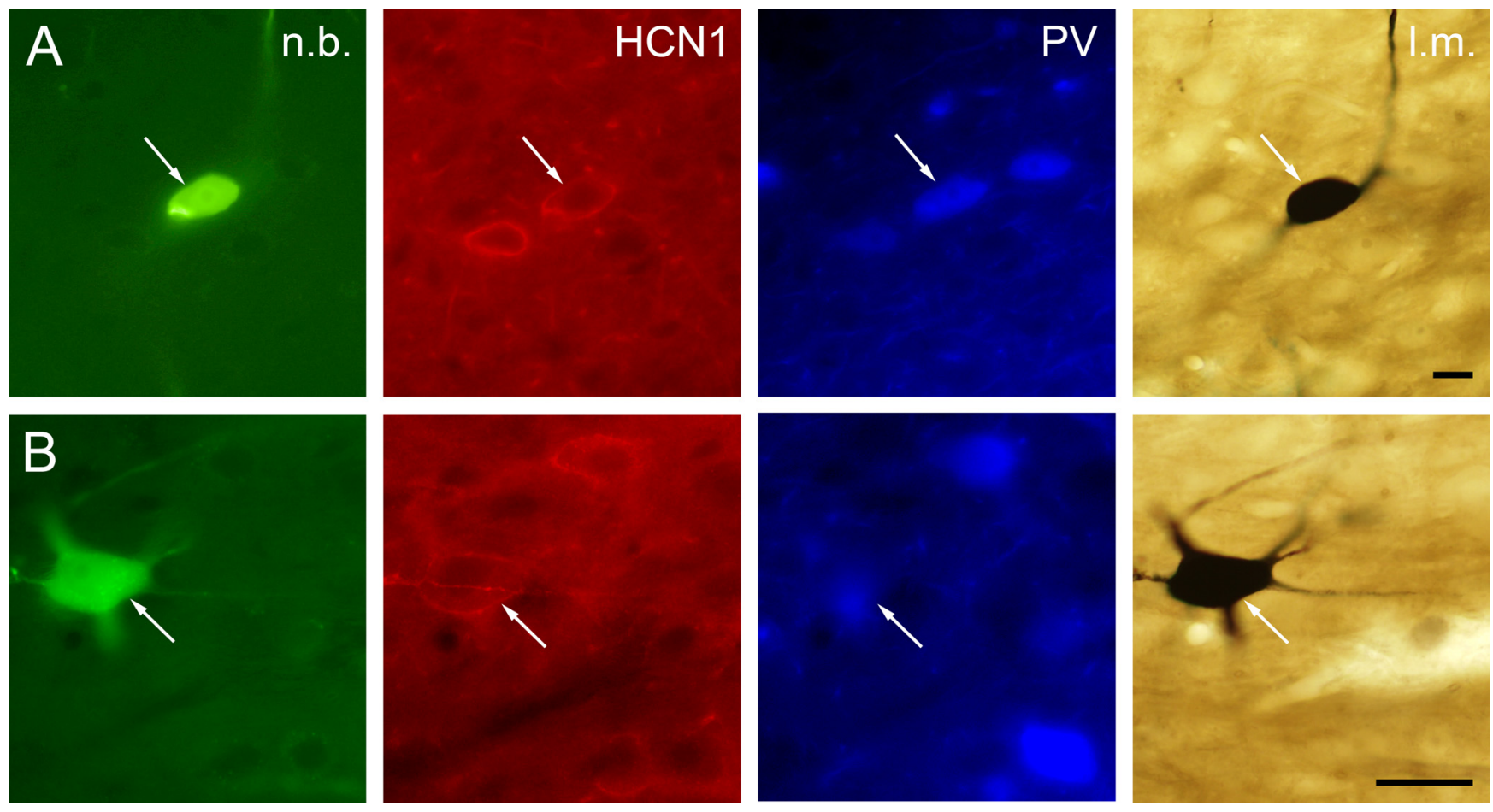

Figure 3. Immunocytochemical identification of juxtacellularly labeled MS neurons. Columns from left, Neurobiotin-labeled soma and/or dendrites; HCN1 immunoreactivity; PV immunoreactivity; light micrograph after nickel-intensified DAB reaction. $\boldsymbol{A}$, The white arrows point to a medial septal neuron expressing both HCN1 and PV. $\boldsymbol{B}$, Representative example of an MS neuron containing HCN1 but not PV (the immunoreactivity near the arrowhead belongs to a cell out of focus). PV-immunoreactive elements can also be observed in the vicinity of the labeled cell. n.b., Neurobiotin; I.m., light micrograph. Scale bars, $10 \mu \mathrm{m}$.

Table 1. Median time lags between identified neuronal groups and hippocampal LFP

\begin{tabular}{lcr}
\hline & Median (ms) & $N$ \\
\hline PV + & 79 & 25 \\
PV - & 11.5 & 16 \\
HCN + & 79 & 20 \\
HCN - & -78 & 7 \\
All & 58 & 189 \\
HC & 47 & 27 \\
\hline
\end{tabular}

All, All medial septal neurons including identified and unidentified cells; $\mathrm{HC}$, putative hippocampal interneurons.

shown to precede hippocampal theta share different amounts of information with hippocampal LFP compared with immunonegative cells, MI during the longest theta and nontheta segments of recorded cells from these anatomical groups was calculated. Eighty-one cells were subjected to this analysis [ $n=18$ PV-IR ( 9 of 18 immunopositive for HCN as well), 11 non-PV, 15 HCN-IR ( 9 of 15 immunopositive for PV as well), 4 non-HCN; 50 unidentified; for the overlap between these groups, see supplemental Table 1, available at www.jneurosci.org as supplemental material]. First, MI values during hippocampal theta were found to be significantly higher than those during nontheta episodes because of a substantial theta-associated elevation of MI $\left(p=5.10 \times 10^{-37}\right)$ (Fig. 5C) (for details, see supplemental notes, available at www.jneurosci.org as supplemental material). Next, we demonstrated that PV-IR cells shared a significantly higher amount of information with hippocampal LFP than PVlacking neurons during theta episodes $\left[p=6.52 \times 10^{-5} ; n=\right.$ 15 PV-IR vs 9 non-PV; neurons with higher than control MI during theta ( $p=0.01$, Wilcoxon's signed rank test, random Poisson control) (see Materials and Methods and supplemen- tal material, available at www.jneurosci.org) were considered] (Fig. 5A,B). Similarly, identified HCN-IR cells were proved to be significantly more synchronous to hippocampal LFP than HCN-immunonegative neurons $(p=0.0176, n=13 \mathrm{HCN}$-IR vs 3 non-HCN). MI values from the anatomically heterogeneous population of unidentified MS neurons were distributed over a wide range with a median between PV (HCN)-IR cells and PV (HCN)-immunonegative neurons. During nontheta epochs, only five neurons showed MI significantly higher than control, from which one was identified as PV-IR. Because theta-bursting neurons were found to show higher mutual information compared with nonbursting cells (Fig. 5D) (supplemental material, available at www.jneurosci.org), one might hypothesize that the theta-related difference between PV (HCN)-IR and PV (HCN)-immunonegative neurons could be caused by the higher number of theta-bursting neurons in the immunopositive groups. Surprisingly, statistically significant difference in MI was detectable between thetabursting PV (HCN)-IR and theta-bursting immunonegative neurons, suggesting that higher synchrony with hippocampal theta is indicative of the above anatomical groups $(p=$ 0.00481 for 14 PV-IR and 5 PV-immunonegative cells; $p=$ 0.00893 for $11 \mathrm{HCN}-\mathrm{IR}$ and $1 \mathrm{HCN}$-immunonegative cell) (Fig. 5B, right).

To compare the extent of synchronization of MS versus hippocampal units to hippocampal theta oscillation, we calculated mutual information for the longest theta and nontheta segments of seven putative hippocampal interneurons as well. Similar to MS neurons, MI values derived from theta episodes were significantly higher than MI values of nontheta segments ( $p=0.0261)$ (Fig. 5E). However, theta-associated MI values of the hippocampal neurons did not reach the level of similar values from medial 
A

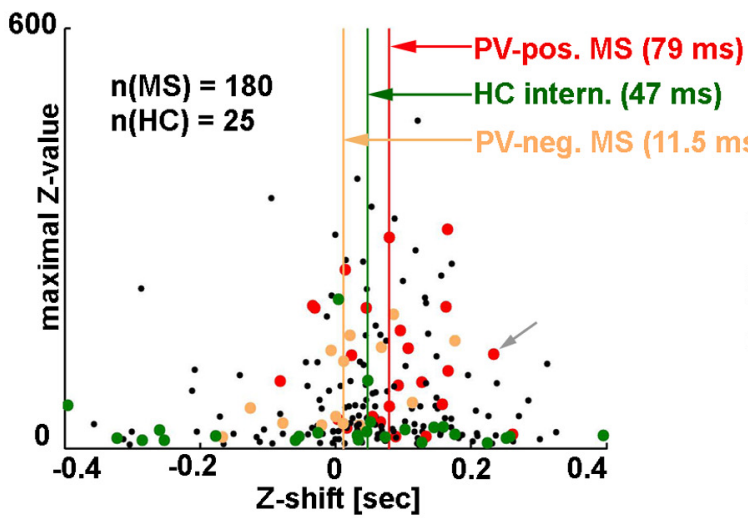

B

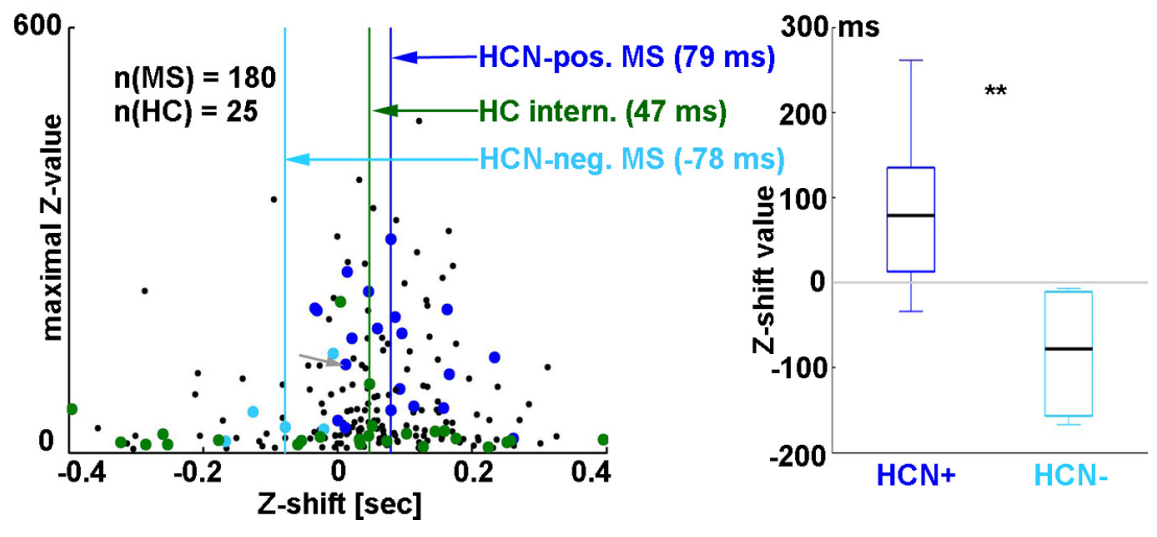

Figure 4. Activity of PV/HCN-IR neurons precedes hippocampal LFP activity. The left plots present summary results of Z-shift analysis of septal and hippocampal neurons showing theta segments on Z-shift ( $x$-axis) versus maximal $Z$ value ( $y$-axis) plane (every point corresponds to the longest theta segment of a recording). $A$, Red, Identified PV-IR septal neurons; orange, PVimmunonegative septal neurons; black, unidentified septal cells; green, putative hippocampal interneurons. The color-coded vertical lines indicate medians of Z-shift values for identified septal anatomical groups and putative hippocampal interneurons. The gray arrow points to data of the neuron shown in Figure 3A. Most of the points corresponding to septal cells (red, orange, and black) fell on the positive halfplane, indicating that changes in their activity preceded hippocampal LFP. Right, Comparison of Z-shift values calculated for PV-IR and PV-immunonegative MS neurons. Septal PV-IR neurons showed significantly higher Z-shift values. $\boldsymbol{B}$, Left, Dark blue, Identified HCN-IR cells; light blue, HCN-immunonegative neurons; black, unidentified MS neurons; green, putative hippocampal interneurons; gray arrow, data from the neuron presented in Figure $3 B$. HCN-IR MS cells preceded putative hippocampal interneurons and HCN-immunonegative cells. Right, Comparison of Z-shift distributions for HCNimmunopositive and HCN-immunonegative neurons. Z-shift values for HCN-IR neurons were significantly higher compared with the values for the $\mathrm{HCN}$-immunonegative neurons. Some points fell out of the range of the left plots presented on this figure ( $9 \mathrm{MS}$ neurons and 2 hippocampal cells); supplemental Figure $2 A-C$ (available at www.jneurosci.org as supplemental material) shows the Z-shift values of all analyzed cells. ${ }^{*} p<0.05 ;{ }^{* *} p<0.01$.

septal cells. Again, we compared mutual information between the two signals in the case of one theta-bursting and two nonbursting hippocampal interneurons. It was found that the theta-bursting hippocampal neuron was more synchronous to the ongoing local field activity than nonbursting cells ( $p=0.00404$ ) (Fig. $5 F$ ) [only units with MI significantly higher than random Poisson control ( $p=0.01$, Wilcoxon's signed rank test) were included] (see Materials and Methods and supplemental material, available at www. jneurosci.org). Nevertheless, MI from the theta-bursting hippocampal cell was lower compared with MI values from MS neurons with bursting activity.

\section{Discussion}

Here, we demonstrated that the activity of the medial septal neurons containing parvalbumin and/or the pacemaker channel $\mathrm{HCN}$ precedes corresponding hippocampal field events. Impor- tantly, this temporal antecedence was still clearly detectable when these putative pacemaker neurons were compared with hippocampal interneurons: the activity of the latter group also preceded LFP changes, but by a 32 ms shorter period compared with the PV/HCN-IR neurons, implying a PV/HCN-IR MS neuron-hippocampal interneuron-pyramidal neuron sequence of influence during septohippocampal theta genesis. Moreover, the above neurons were found to share a high amount of information with the ongoing hippocampal field oscillation during theta rhythm, reflecting the central role of these cells in the theta-associated septohippocampal synchronization process.

The medial septum was proved to be a key determinant of hippocampal theta genesis (Vertes and Kocsis, 1997). However, both experimental (Tóth et al., 1993) and modeling (Wang, 2002) studies have shown that, in addition to the septal drive, hippocampo-septal feedback is also essential for the generation and maintenance of hippocampal theta oscillation. Moreover, the classical view of medial septal theta generation has recently been challenged by in vitro data (Manseau et al., 2008), suggesting a hypothesis of hippocampal lead over the medial septum in the regulation of theta. The cited study showed that even the fundamental question regarding the source of theta rhythmic drive in the septo-hippocampal system is still not resolved.

In our previous studies, we identified two overlapping groups of MS neurons with anatomical and electrophysiological features ideal for pacemakers of theta rhythm in the septo-hippocampal system (Borhegyi et al., 2004; Varga et al., 2008). To investigate whether these MS cell groups may function as the sources of theta rhythmic drive to the hippocampus, novel analysis tools had to be introduced to examine their relationship with the hippocampal network. Therefore, by using the recordings of these previously identified neurons in combination with hippocampal unit recording, we aimed to provide clues about their position in the process of septo-hippocampal theta genesis. Since the relationship of identified MS neurons to hippocampal activity was in the focus of our study and single-cell labeling is still not possible at sufficiently high success rate in anesthetic-free animals, recordings were made under urethane anesthesia. In addition to acknowledging the difference between natural and urethane-induced sleep (Leung, 1985; Simon et al., 2006), several characteristics of the latter including the spontaneous alteration of theta and nontheta state can be observed under urethane anesthesia (Clement et al., 2008).

First in the analysis process, we aimed to reconstruct the temporal pattern of septo-hippocampal activity changes during theta formation by implementing the recently developed $Z$-shift 
method (Siapas et al., 2005). The observed 79 ms delay between the unit activity of septal HCN- and/or PV-immunoreactive neurons and hippocampal field oscillation could reflect the time taken by the transmission of the rhythmic output of MS GABAergic pacemaker neurons to hippocampal interneurons that ultimately results in synchronization of the hippocampal network and a detectable field oscillation. Importantly, the $47 \mathrm{~ms}$ time delay between the hippocampal interneuron activity and LFP reflects (1) the delayed activation of interneurons compared with PV/HCN-IR MS cells (47 vs $79 \mathrm{~ms}$ before LFP) and (2) the antecedence of interneuron activity changes compared with hippocampal pyramidal cells. However, it should be noted that, although the majority of MS cells (139 of 189) appeared to precede hippocampal theta, a smaller population (50 of 189) (see Results) showed negative $Z$-shift values (median, $-78 \mathrm{~ms}$ ). This group (6 PV-negative/HCN-negative; 2 PV-positive/HCN-positive; $1 \mathrm{PV}$ positive/not tested for $\mathrm{HCN}$; 1 PV-negative/HCN-positive; 40 unidentified) was characterized by weaker phase coupling (i.e., lower median $Z$ values: 34 vs 54) and lower proportion of bursting neurons (46 vs $58 \%)$. We propose that these neurons form a septal "follower" group, receiving rhythmic input from hippocampal and/or from GABAergic medial septal neurons. They might contribute to the stabilization of synchronous activity by providing a fluctuating inhibitory tone to the pacemaker population.

The time lag between MS GABAergic neurons and hippocampal interneurons is substantially higher than the sum of the conduction time of septo-hippocampal axons ( $<5 \mathrm{~ms}$ ) (Jones et al., 1999), synaptic delays, and latency of action potential genesis (Fricker and Miles, 2000). This finding can be paralleled by previous data showing that MS phasic theta ON neurons change their firing activity $\sim 500 \mathrm{~ms}$ before the LIA to theta transition of the hippocampal EEG (Bland et al., 1999). Furthermore, in a model hippocampal network, delayed synchronization of its component subnetworks of basket, oriens-lacunosum moleculare, and pyramidal cells was demonstrated (Orbán et al., 2006). Thus, the delays in the septohippocampal system may reflect the time necessary for the gradual recruitment of the interconnected groups of neurons leading to the accumulation of synchronously firing oscillators and the appearance of the regular theta field activity. An additional implication of the above scenario is that the perturbation of one of the elements of the septo-hippocampal network will initiate a chain of events taking several tens of milliseconds to be manifested at the population level. It should be noted that Siapas et al. (2005) reported no significant temporal difference between hip-

B
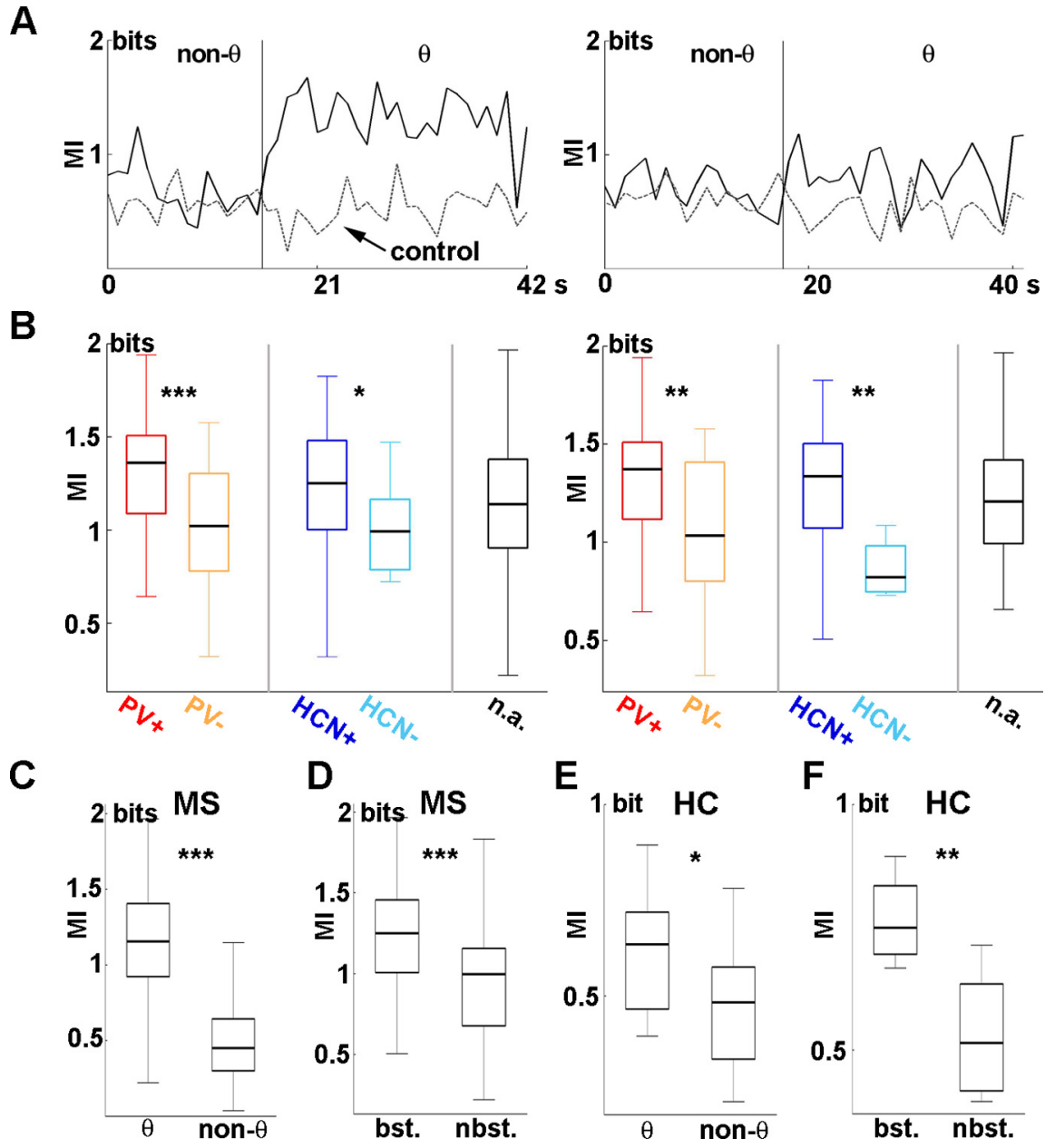

Figure 5. Theta-associated increase of MI between septal PV/HCN-IR neurons and hippocampal LFP. A, Left, Mutual information for a PV/HCN-IR MS neuron. Solid line, MI of real data; dashed line, MI of control. The black vertical line separates the longest theta and (usually nonadjacent) nontheta segment. Note the substantially higher MI during theta compared with nontheta. Right, Real (solid) and control (dashed) MI calculated for a PV/HCN-immunonegative MS neuron. Only a subtle theta-associated increase values compared with HCN-immunonegative cells (light blue). The black box displays the MI distribution from unidentified MS among anatomical groups regarding theta-associated increases of Ml were present despite excluding the nonbursting MS neurons. C, Comparison of MI values during theta and nontheta segments. A significant theta-associated increase could be detected. D, Comparison of MI values calculated for theta segments in theta-bursting and nonbursting MS neurons. Theta bursters showed significantly higher MI. $\boldsymbol{E}, \boldsymbol{F}$, Mutual information was calculated between unit activity of putative interneurons of the hippocampus and hippocampal LFP.E, Comparison of MI during theta and nontheta segments. Significant theta-associated increase of MI could also be detected for hippocampal neurons, albeit MI of putative hippocampal interneurons did not reach the level of MS cells during theta oscillation (compare the different scaling of $\boldsymbol{C}$ and $\boldsymbol{E}$ ). $\boldsymbol{F}$, Comparison of bursting and nonbursting hippocampal cells. Theta-bursting neurons showed significantly higher MI compared with nonbursting cells. HC, Hippocampus; bst., bursting; nbst., nonbursting. ${ }^{*} p<0.05$; ${ }^{* *} p<0.01$; ${ }^{* *} p<0.001$.

pocampal unit and field activity, which was probably attributable to their mixed sample of pyramidal cells and interneurons. Hippocampal interneurons form an extremely heterogeneous cell population (Freund and Buzsáki, 1996). Various subgroups of interneurons were shown to fire at different phases of hippocampal theta field oscillation (Somogyi and Klausberger, 2005) and to be differentially innervated by medial septal neurons (Eyre et al., 2007). This diversity could account for the observed wide range of time lags for putative hippocampal interneurons as well as for the lower median MI values of hippocampal interneurons when compared with MS cells. Thus, the differential contribution of anatomically identified interneuron subgroups to theta genera- 
tion needs to be further investigated on a larger sample of hippocampal cells.

In the second part of our analysis, differential contribution of anatomically identified medial septal cell groups to septohippocampal information transmission has been assessed. A well established information theoretical approach using entropy, the classical measure of information content (Shannon, 1948), was chosen, based on its ability to detect both the linear and nonlinear aspects of synchronization (Kajikawa and Hackett, 2005). Entropy was applied to wavelet spectra of hippocampal field potentials and unit recordings, for calculating the information content of time-frequency wavelet windows in the theta band [wavelet entropy (Yordanova et al., 2002)]. The application of wavelet entropy allowed us to bridge the qualitative difference of the LFP and single-unit signal. Also, decomposing the data in the frequency domain through wavelet calculation made us capable of investigating theta band separately. Importantly, theta band was selected because (1) the medial septum principally affects the hippocampus through the generation of theta rhythm; (2) information in other frequency bands was relatively sparse during theta segments, whereas the theta band still carried considerable amount of information during nontheta states (Fig. $2 B$ ). The high amount of shared information between $\mathrm{HCN} / \mathrm{PV}$-neurons and hippocampal LFP compared with non-PV/non-HCN or with hippocampal interneurons indicates dominant contribution of the former MS cell groups to hippocampal theta genesis over the latter neurons.

On the course of theta synchronization, septo-hippocampal GABAergic pacemaker neurons inhibit all known subsets of hippocampal interneurons, which leads to the disinhibition of pyramidal cells (Freund and Antal, 1988; Tóth et al., 1997) (Fig. 6). The oscillating output of the hippocampal interneuron network produces the alternating charge flow along the soma-dendritic axis of pyramidal cells leading to the generation of field theta activity. Hippocampo-septal backprojection, targeting mostly septo-hippocampal GABAergic cells, would further enhance synchrony of MS GABAergic cells (Tóth et al., 1993; Takács et al., 2008). The above mutual inhibitory loop forms the core of the theta generating circuitry. In contrast with the GABAergic MS cells innervating interneurons exclusively, septal cholinergic neurons send diffuse projection to the hippocampus, influencing both interneurons and principal cells (Frotscher and Léránth, 1985). Selective lesion of the medial septal cholinergic cells strongly reduces the number of rhythmically bursting neurons in the MS (Apartis et al., 1998) and attenuates hippocampal field theta (Yoder and Pang, 2005). However, a recent study by Simon et al. (2006) demonstrated that anatomically identified cholinergic MS neurons display very slow, theta-unrelated firing in vivo. This suggests that these neurons might not act as pacemakers but rather play a permissive and modulatory role via the tonic excitation of MS GABAergic and glutamatergic neurons (Wu et al., 2000; Manseau et al., 2005) as well as hippocampal interneurons and principal cells (Frotscher and Léránth, 1985). Additionally, abrupt changes of cholinergic activity can also contribute to the resetting or blocking of the oscillation via ionotropic acetylcholine receptors. Recently, a substantial proportion of septal neurons have been shown to be glutamatergic (Sotty et al., 2003). These cells innervate septal GABAergic and cholinergic neurons (Hajszan et al., 2004; Manseau et al., 2005), display slow firing or cluster firing discharge pattern in vitro (Sotty et al., 2003), and may also project to the hippocampus. These data suggest that septal glutamatergic neurons may be involved in theta generation, which is also supported by a recent theoretical study (Uj-
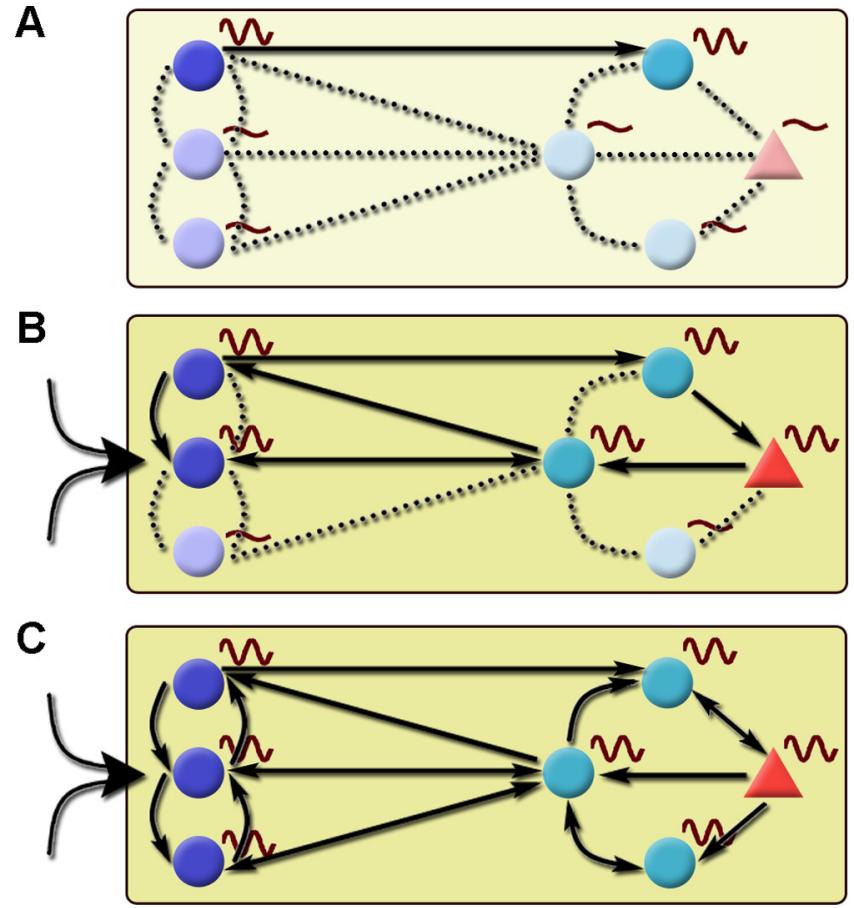

Figure 6. Gradual recruitment of oscillating units in the septo-hippocampal system leads to population level theta rhythm. In general, theta activity within the septo-hippocampal system is formed by the buildup of synchrony among groups of interconnected oscillating units and spreads by recruiting nonoscillating components of the network. Reciprocal GABAergic inhibitory interactions under the control of tonic neuromodulatory action (muscarinic cholinergic and monoaminergic) and phasic rapid excitation (nicotinic cholinergic and glutamatergic) is indispensable for the operation of this rhythm-generating circuitry. Left side, MS; right side, hippocampus. Circle, GABAergic; triangle, pyramidal cell. $A$, During nontheta states, only a subset of medial septal and hippocampal neurons exhibit theta frequency membrane potential oscillation [dark colored cells with sine waves; in the MS, the so-called constitutive bursting cells in the studies by Borhegyi et al. (2004) and Varga et al. (2008)]. These oscillating units are only partly synchronized; most of them are functionally uncoupled. The out-of-phase activity can even weaken population-level synchrony. $\boldsymbol{B}$, The elevation of ascending excitation (large arrow left to MS cells) pushes the septo-hippocampal system into a short transition state characterized by the abrupt elevation of GABAergic activity, the activation of pacemaker mechanisms, and the rapid spread of synchronous activity via the coupling of activated medial septal and hippocampal oscillators. Tonic excitation of the septo-hippocampal GABAergic circuitry by septal cholinergic neurons (indicated by yellow background color of variable intensity) also contributes to this process. The reciprocal septo-hippocampal dialogue has a crucial role not only in connecting the two regions but also in orchestrating rhythmic units within them. $C$, The spread of synchrony leads to the formation of population level rhythm. The gradual buildup of oscillation is supported by the variance of temporal antecedence observed in this study: some rhythmic units precede hippocampal LFP more than others, indicating that they might be involved in an earlier phase of the synchronization process. The overlap of MS and hippocampal Z-shift values also points to the importance of the two-way interaction between the MS and hippocampus for both the formation and maintenance of the oscillation.

falussy and Kiss, 2006). However, the in vivo activity pattern of MS glutamatergic cells and their exact role in theta genesis remain to be established. Thus, theta genesis in the septo-hippocampal reciprocal inhibitory loop may be triggered by excitatory inputs that convey incoming sensory load and cortical influence. Excitatory elements are also capable of setting the frequency and amplitude of the oscillation (Ujfalussy and Kiss, 2006).

\section{References}

Apartis E, Poindessous-Jazat FR, Lamour YA, Bassant MH (1998) Loss of rhythmically bursting neurons in rat medial septum following selective lesion of septo-hippocampal cholinergic system. J Neurophysiol 79:1633-1642.

Bland BH, Oddie SD, Colom LV (1999) Mechanisms of neural synchrony in 
the septohippocampal pathways underlying hippocampal theta generation. J Neurosci 19:3223-3237.

Borhegyi Z, Varga V, Szilágyi N, Fabo D, Freund TF (2004) Phase segregation of medial septal GABAergic neurons during hippocampal theta activity. J Neurosci 24:8470-8479.

Buzsáki G, Leung LW, Vanderwolf CH (1983) Cellular bases of hippocampal EEG in the behaving rat. Brain Res 287:139-171.

Clement EA, Richard A, Thwaites M, Ailon J, Peters S, Dickson CT (2008) Cyclic and sleep-like spontaneous alternations of brain state under urethane anaesthesia. PLoS ONE 3:e2004.

Csicsvari J, Hirase H, Czurkó A, Mamiya A, Buzsáki G (1999) Oscillatory coupling of hippocampal pyramidal cells and interneurons in the behaving rat. J Neurosci 19:274-287.

Dekhuijzen AJ, Bagust J (1996) Analysis of neural bursting: nonrhythmic and rhythmic activity in isolated spinal cord. J Neurosci Methods 67:141-147.

Eyre MD, Freund TF, Gulyas AI (2007) Quantitative ultrastructural differences between local and medial septal GABAergic axon terminals in the rat hippocampus. Neuroscience 149:537-548.

Fisher N (1993) Statistical analysis of circular data. Cambridge, UK: Cambridge UP.

Freiwald WA, Valdes P, Bosch J, Biscay R, Jimenez JC, Rodriguez LM, Rodriguez V, Kreiter AK, Singer W (1999) Testing non-linearity and directedness of interactions between neural groups in the macaque inferotemporal cortex. J Neurosci Methods 94:105-119.

Freund TF, Antal M (1988) GABA-containing neurons in the septum control inhibitory interneurons in the hippocampus. Nature 336:170-173.

Freund TF, Buzsáki G (1996) Interneurons of the hippocampus. Hippocampus 6:347-470.

Fricker D, Miles R (2000) EPSP amplification and the precision of spike timing in hippocampal neurons. Neuron 28:559-569.

Frotscher M, Léránth C (1985) Cholinergic innervation of the rat hippocampus as revealed by choline acetyltransferase immunocytochemistry: a combined light and electron microscopic study. J Comp Neurol 239:237-246.

Fuentealba P, Begum R, Capogna M, Jinno S, Márton LF, Csicsvari J, Thomson A, Somogyi P, Klausberger T (2008) Ivy cells: a population of nitricoxide-producing, slow-spiking GABAergic neurons and their involvement in hippocampal network activity. Neuron [Erratum (2008) 58:295] 57:917-929.

Hajszan T, Alreja M, Leranth C (2004) Intrinsic vesicular glutamate transporter 2-immunoreactive input to septohippocampal parvalbumincontaining neurons: novel glutamatergic local circuit cells. Hippocampus 14:499-509.

Hurtado JM, Rubchinsky LL, Sigvardt KA (2004) Statistical method for detection of phase-locking episodes in neural oscillations. J Neurophysiol 91:1883-1898.

Jones GA, Norris SK, Henderson Z (1999) Conduction velocities and membrane properties of different classes of rat septohippocampal neurons recorded in vitro. J Physiol 517:867-877.

Kajikawa Y, Hackett TA (2005) Entropy analysis of neuronal spike train synchrony. J Neurosci Methods 149:90-93.

Klausberger T, Magill PJ, Márton LF, Roberts JD, Cobden PM, Buzsáki G, Somogyi P (2003) Brain-state- and cell-type-specific firing of hippocampal interneurons in vivo. Nature 421:844-848.

Lawson VH, Bland BH (1993) The role of the septohippocampal pathway in the regulation of hippocampal field activity and behavior: analysis by the intraseptal microinfusion of carbachol, atropine, and procaine. Exp Neurol 20:132-144.

Leung LW (1985) Spectral analysis of hippocampal EEG in the freely moving rat: effects of centrally active drugs and relations to evoked potentials. Electroencephalogr Clin Neurophysiol 60:65-77.
Manseau F, Danik M, Williams S (2005) A functional glutamatergic neurone network in the medial septum and diagonal band area. J Physiol 566:865-884

Manseau F, Goutagny R, Danik M, Williams S (2008) The hippocamposeptal pathway generates rhythmic firing of GABAergic neurons in the medial septum and diagonal bands: an investigation using a complete septohippocampal preparation in vitro. J Neurosci 28:4096-4107.

Orbán G, Kiss T, Erdi P (2006) Intrinsic and synaptic mechanisms determining the timing of neuron population activity during hippocampal theta oscillation. J Neurophysiol [Erratum (2007) 97:1869] 96:2889-2904.

Panzeri S, Senatore R, Montemurro MA, Petersen RS (2007) Correcting for the sampling bias problem in spike train information measures. J Neurophysiol 98:1064-1072.

Shannon C (1948) A mathematical theory of communication. Bell Syst Tech J 27:379-423, 623-656.

Siapas AG, Lubenov EV, Wilson MA (2005) Prefrontal phase locking to hippocampal theta oscillations. Neuron 46:141-151.

Simon AP, Poindessous-Jazat F, Dutar P, Epelbaum J, Bassant MH (2006) Firing properties of anatomically identified neurons in the medial septum of anesthetized and unanesthetized restrained rats. J Neurosci 26:9038-9046.

Somogyi P, Klausberger T (2005) Defined types of cortical interneurone structure space and spike timing in the hippocampus. J Physiol 562:9-26.

Sotty F, Danik M, Manseau F, Laplante F, Quirion R, Williams S (2003) Distinct electrophysiological properties of glutamatergic, cholinergic and GABAergic rat septohippocampal neurons: novel implications for hippocampal rhythmicity. J Physiol 551:927-943.

Takács VT, Freund TF, Gulyás AI (2008) Types and synaptic connections of hippocampal inhibitory neurons reciprocally connected with the medial septum. Eur J Neurosci 28:148-164.

Torrence C, Compo GP (1998) A practical guide to wavelet analysis. Bull Am Meteorol Soc 79:61-78.

Tóth K, Borhegyi Z, Freund TF (1993) Postsynaptic targets of GABAergic hippocampal neurons in the medial septum-diagonal band of Broca complex. J Neurosci 13:3712-3724.

Tóth K, Freund TF, Miles R (1997) Free in PMC disinhibition of rat hippocampal pyramidal cells by GABAergic afferents from the septum. J Physiol 500:463-474.

Ujfalussy B, Kiss T (2006) How do glutamatergic and GABAergic cells contribute to synchronization in the medial septum? J Comput Neurosci 21:343-357.

Varga V, Hangya B, Kránitz K, Ludányi A, Zemankovics R, Katona I, Shigemoto R, Freund TF, Borhegyi Z (2008) The presence of pacemaker HCN channels identifies theta rhythmic GABAergic neurons in the medial septum. J Physiol 586:3893-3915.

Vertes RP, Kocsis B (1997) Brainstem-diencephalo-septohippocampal systems controlling the theta rhythm of the hippocampus. Neuroscience 81:893-926.

Wang XJ (2002) Pacemaker neurons for the theta rhythm and their synchronization in the septohippocampal reciprocal loop. J Neurophysiol 87:889-900.

Wu M, Shanabrough M, Leranth C, Alreja M (2000) Cholinergic excitation of septohippocampal GABA but not cholinergic neurons: implications for learning and memory. J Neurosci 20:3900-3908.

Yoder RM, Pang KC (2005) Involvement of GABAergic and cholinergic medial septal neurons in hippocampal theta rhythm. Hippocampus 15:381-392.

Yordanova J, Kolev V, Rosso OA, Schürmann M, Sakowitz OW, Ozgören M, Basar E (2002) Wavelet entropy analysis of event-related potentials indicates modality-independent theta dominance. J Neurosci Methods 117: 99-109. 